\title{
Variation spatiotemporelle de la cryptorchidie et de l'hypospadias au Québec : Une étude exploratoire
}

\author{
Sophie Guertin; ${ }^{*}$ Karine-Sylvie Lemieux; ${ }^{*}$ Natalie Makhoulian; ${ }^{*}$ Sébastien Michaud; ${ }^{*}$ Rose-Marie Patry ${ }^{*}$ \\ Anne-Andrée Côté, MD; Fabien Gagnon, MD, FRCPC; ${ }^{*}$ Pierre Ayotte, PhD; ${ }^{\text {t\$ }}$ Stéphane Bolduc, MD, FRCSC ${ }^{\text {st }}$
}

"Faculté de médecine et des sciences de la santé, Université de Sherbrooke, Sherbrooke, QC; †'Institut national de santé publique du Québec, Montréal, QC; §Faculté de médecine, Université Laval, Québec, QC; 土Département d'urologie pédiatrique, Centre hospitalier universitaire de Québec, Pavillon CHUL, Québec, QC

See related article on page 172 .

Cite as: Can Urol Assoc J 2011;5(3):167-71; D01:10.5489/cuaj.10090

\section{Résumé}

Objectifs : La majorité des études effectuées au cours des dernières décennies ont mis en évidence une augmentation du nombre de cas de cryptorchidie et d'hypospadias entre 1970 et 1990. De plus, I'importante variabilité géographique de ces anomalies est bien décrite. Cette étude vise à mesurer la prévalence à la naissance de la cryptorchidie et de I'hypospadias au Québec, à vérifier si ces anomalies sont en augmentation et à en évaluer la répartition interrégionale.

Méthode : Une étude épidémiologique descriptive a été réalisée à partir du nombre de garçons de cinq ans et moins hospitalisés pour une cryptorchidie ou un hypospadias au Québec de 1989 à 2004 selon les données du fichier administratif d'hospitalisation MEDÉCHO. Les données sur les naissances provenaient de l'Institut de la statistique du Québec.

Résultats : La prévalence annuelle moyenne pour 1000 naissances vivantes de sexe masculin est de 19,1 (IC à $95 \%$ : 18,8-19,4) pour la cryptorchidie et 11,4 (IC à $95 \%$ 11,1-11,6) pour I'hypospadias au Québec. Au cours de la période étudiée, la prévalence de cryptorchidie a légèrement diminué, alors que celle de I'hypospadias est demeurée stable. Comparativement à la province du Québec, des régions présentent une prévalence significativement différente de cryptorchidie et/ou d'hypospadias. .

Conclusion : Au Québec, la prévalence de cryptorchidie est en légère diminution alors que celle de l'hypospadias est stable. Des variations régionales significatives sont observables. D'autres études sont nécessaires afin d'évaluer l'hypothèse d'un lien avec les contaminants environnementaux en émergence. L'implantation d'un système de surveillance des anomalies congénitales permettrait une représentation plus valide de la situation.

\section{Abstract}

Objectives: Previous research has shown evidence of an increase in the number of cases of cryptorchidism and hypospadias between 1970 and 1990. Geographical disparities of these anomalies are widely described. This study aims to measure the prevalence of cryptorchidism and hypospadias at birth in the province of Quebec, to investigate if there is an increasing trend and to assess the interregional distribution of these anomalies.

Method: A descriptive epidemiological study was undertaken to investigate the number of newborn males up to five years of age, hospitalized for cryptorchidism or hypospadias in Quebec between 1989 and 2004 based on data collected from MED-ECHO, a database compiling hospitalizations and used for administrative purposes. Birth rates were provided by the Statistical Institute of Quebec. Results: Mean yearly prevalence per 1000 male live births was 19.1 (95\% Cl 18.8-19.4) for cryptorchidism and $11.4(95 \% \mathrm{Cl}$ 11.1-11.6) for hypospadias for the province of Quebec. Within the period of study, the prevalence of cryptorchidism decreased slightly while that of hypospadias remained stable. Significant variations in prevalence were observed in some regions compared to the province, for both pathologies.

Conclusion: In Quebec, the prevalence of cryptorchidism is decreasing while that of hypospadias is stable. There is significant regional variation among the province. More studies are needed to assess the potential link with environmental contaminants as an emerging explanation. The implementation of an adequate surveillance system for congenital anomalies would allow for a more accurate representation of the situation.

\section{Introduction}

La descente testiculaire fotale se divise en deux étapes. Elle débute entre la $8^{\mathrm{e}}$ et la $15^{\mathrm{e}}$ semaine de gestation par le transit intra-abdominal, contrôlé principalement par l'hormone insulin-like 3 (INSL3) et son récepteur couplé à la protéine-G riche en motifs leucine répétée 8 (LGR8), et se poursuit au cours du $7^{\mathrm{e}}$ mois de gestation par la migration inguinoscrotale sous influence hormonale androgénique ${ }^{1}$. La virili- 
sation des organes génitaux externes s'effectue à partir de la $8^{\mathrm{e}}$ semaine et nécessite la sécrétion de testostérone et de dihydrotestostérone par les cellules de Leydig².

Un défaut $d^{\prime}$ embryogenèse peut mener à une cryptorchidie et/ou un hypospadias. Parmi les facteurs de risque reconnus pour les deux anomalies, on retrouve la prématurité ${ }^{3-6}$, le faible poids pour l'âge gestationne $\left.\right|^{3,5-7}$, le faible poids à la naissance ${ }^{4,5,7-10}$ et la faible parité, ${ }^{4,8-10}$. Le tabagisme maternel pourrait être associé à la cryptorchidie ${ }^{8}$, alors que l'exposition de la mère à la fumée secondaire pourrait être associée à I'hypospadias ${ }^{3,6}$. On a également rapporté un risque accru de cryptorchidie en présence de diabète gestationnel ${ }^{11}$ alors que I'hypospadias a été associé à un diabète préexistant ${ }^{12}$ et à une hypertension gestationnelle ${ }^{3,8}$. De plus, la présence de la même anomalie chez un frère plus âgé augmente le risque de cryptorchidie ( $R C=3,84$ ) et d'hypospadias ( $R C$ $=10,08$ ) chez un enfant ${ }^{10}$. Les anomalies chromosomiques telles que les syndromes de Down, de Prune Belly et de Prader-Willi sont également associées à un risque accru de cryptorchidie ${ }^{1}$.

Toutefois, ces facteurs ne peuvent expliquer à eux seuls I'importante variabilité géographique de la prévalence à l'échelle mondiale, allant de 0,03 à 13,4\% pour la cryptorchidie et de 0,05 à $0,4 \%$ pour I'hypospadias ${ }^{13}$. De plus, la majorité des études effectuées au cours des dernières décennies ont mis en évidence une augmentation du nombre de cas entre 1970 et $1990^{1,13-16}$, mais les données demeurent divergentes quant à l'évolution récente de ces anomalies ${ }^{1,7,12}$.

On tente $d^{\prime}$ expliquer ces phénomènes par la présence de perturbateurs endocriniens environnementaux (PEE), définis comme des "substances exogènes qui interfèrent avec la synthèse, le transport, la liaison, l'action ou l'élimination des hormones endogènes responsables du maintien de l'homéostasie, de la reproduction, du développement et/ ou du comportement ${ }^{17,18}$. La conséquence d'une action hormonale anti-androgénique ou œestrogénique sur le système reproducteur mâle en développement se manifeste par une immaturité des cellules germinales testiculaires fœtales. Ceci serait à l'origine du syndrome de dysgénésie testiculaire (SDT) regroupant la diminution de la qualité du sperme, la cryptorchidie, I'hypospadias et le cancer testiculaire ${ }^{2,4,19-22}$. Parmi les PEE incriminés, on retrouve les herbicides (p. ex. linuron, procymidone, lindane) ${ }^{17,18,23}$, les fongicides (p. ex. méthoxychlore) $)^{17,18,24}$, les insecticides (p. ex. DDT, aldrin et dieldrin) ${ }^{4,17,18,23-25}$, les phtalates (plastifiants présents dans les cosmétiques et certains jouets pour

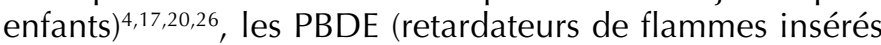
dans le plastique et les produits textiles) ${ }^{17,27}$ et les BPC (autrefois utilisés dans les huiles électriques, les lubrifiants, les adhésifs et les peintures) $)^{4,17,18}$.

Cette étude vise donc à mesurer la prévalence à la naissance de la cryptorchidie et de I'hypospadias au Québec, à vérifier si ces anomalies sont en augmentation et à en évaluer la répartition spatiale à l'échelle de la province.

\section{Méthodologie}

Une étude épidémiologique descriptive a été réalisée à partir du nombre de garçons de cing ans et moins hospitalisés pour une cryptorchidie ou un hypospadias au Québec de 1989 à 2004, en relevant leur région sociosanitaire (RSS) de résidence. Les données ont été extraites de MED-ÉCHO, un fichier administratif d'hospitalisation à participation obligatoire qui contient l'information sur toutes les hospitalisations et les chirurgies d'un jour survenues dans tous les hôpitaux de soins aigus du Québec depuis le $1^{\text {er }}$ avril $1981^{28}$. Les cas ont été repérés à l'aide de la codification du classement international des maladies 9 (CIM-9). Les codes 752,5 pour la cryptorchidie et 752,6 pour I'hypospadias ont été recherchés dans le diagnostic principal et les 15 diagnostics secondaires apparaissant dans le dossier d'hospitalisation MED-ÉCHO. Afin que les enfants hospitalisés à plusieurs reprises pour le même problème soient comptés comme un seul cas, une clé d'appariement a été utilisée, composée du numéro de dossier hospitalier, du sexe et de la RSS de résidence. La formule suivante (tableau 1 ) a été utilisée afin de calculer la prévalence à la naissance de cryptorchidie et d'hypospadias pour le Québec et pour chaque RSS :

Le nombre total de nouveau-nés provient des données de naissance de l'Institut de la statistique du Québec ${ }^{29}$. Cette

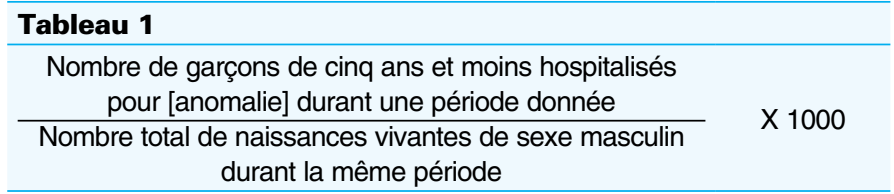

méthode s'apparente à celle déjà utilisée par le ministère de la Santé et des Services sociaux du Québec (MSSS) pour le suivi des anomalies congénitales jugées les plus importantes, courantes et faciles à diagnostiquer ${ }^{30}$.

Les taux calculés sont sujets à une certaine imprécision statistique liée à la rareté relative des diagnostics étudiés. $C^{\prime}$ est pourquoi des taux annuels moyens ont été calculés sur 3 ans pour l'analyse temporelle au niveau du Québec et sur 15 ans pour l'analyse comparative entre les régions sociosanitaires et le Québec. Un intervalle de confiance (IC) à $95 \%$ a été calculé selon une approche binomiale à partir de l'ensemble des cas et des naissances vivantes pour une période donnée.

\section{Résultats}

Pour l'ensemble des périodes triennales à l'étude, on observe au Québec un nombre moyen de cas par année variant entre 696,7 et 963,3 pour la cryptorchidie et entre 407,7 et 553,0 pour l'hypospadias (tableau 2). Il en résulte des taux annuels moyens pour 1000 naissances variant 
entre 18,4 et 20,3 pour la cryptorchidie et entre 10,8 et 11,9 pour I'hypospadias (graphique 1 ). Sur I'ensemble de la période couverte, la prévalence provinciale annuelle moyenne est de 19,1 (IC à $95 \%$ : 18,8-19,4) pour la cryptorchidie (graphique 2) et de 11,4 (IC à $95 \%: 11,1-11,6$ ) pour I'hypospadias (graphique 3).

Dans le cas de la cryptorchidie, les taux annuels moyens ont diminué de façon significative à partir de la période de 1995-96 à 1997-98 comparativement à la première période de 1989-90 à 1991-92, comme en fait foi l'absence de chevauchement des IC à $95 \%$. Les taux annuels moyens d'hypospadias sont plutôt demeurés stables entre 1989 et 2004 (graphique 1).

Enfin, en comparant les différentes régions sociosanitaires à l'ensemble du Québec pour la période étudiée, on observe des variations régionales significatives. Tel qu'en témoigne I'absence de chevauchement des IC à $95 \%$, la prévalence de cryptorchidie est supérieure à celle de la province dans quatre régions (Bas-Saint-Laurent, Capitale-Nationale, Gaspésie-îles-de-la-Madeleine, Chaudière-Appalaches), tandis qu'elle y est inférieure dans trois autres (Estrie, Outaouais, Nunavik) (graphique 2). Pour sa part, la prévalence d'hypospadias est inférieure à celle du Québec dans cinq régions (Saguenay-Lac-Saint-Jean, Estrie, Outaouais, Nunavik, Terres-Cries-de-la-Baie-James) (graphique 3).

\section{Discussion}

Au terme de cette étude, il apparaît que la prévalence annuelle moyenne de la cryptorchidie au Québec est comparable à ce qui a été rapporté ailleurs dans le monde (0,3 à 134 pour 1000 naissances; incluant les cas prématurés) ${ }^{1,13}$. Par contre, la prévalence annuelle moyenne d'hypospadias est supérieure à celle attendue $(0,55$ à 3,97 pour 1000 naissances) $)^{1,13}$. Au Canada, les taux annuels observés pour 1000 naissances sont de 3,65 pour la cryptorchidie et de 2,8 pour I'hypospadias ${ }^{31}$, ce qui est inférieur à ceux du Québec. Ces écarts pourraient s'expliquer par l'inclusion ou non des mortinaissances et des filles dans le nombre de naissances, la variabilité des définitions de $\operatorname{cas}^{31}$ et les différentes méthodes de dénombrement utilisées ${ }^{1,13,15}$. Pour sa part, la banque de données MED-ÉCHO ne comptabilise pas systématiquement les interruptions volontaires de grossesse

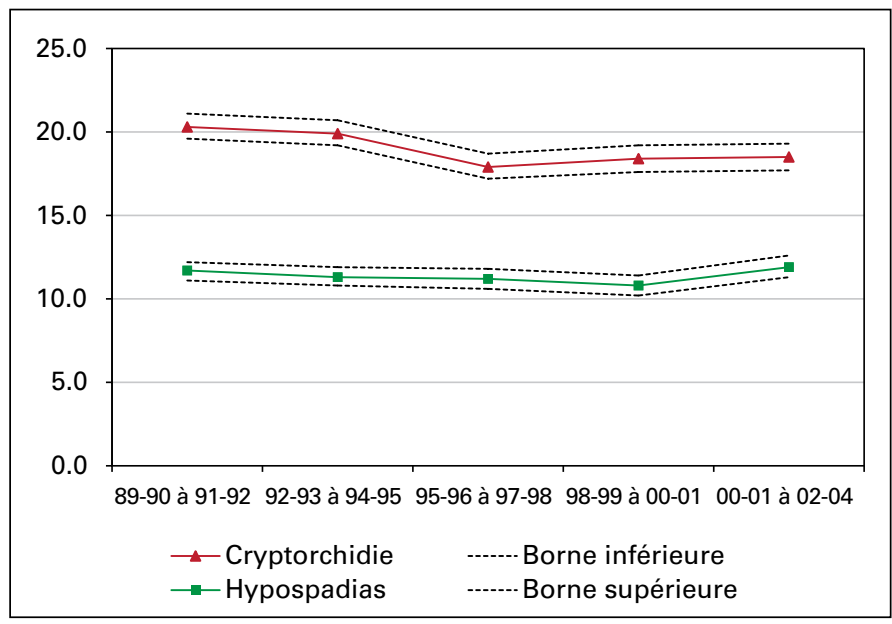

Graphique 1. Taux annuel moyen de cryptorchidie et d'hypospadias pour 1000 naissances vivantes de sexe masculin, Québec, 1989-2004.

(IVG), les avortements spontanés et les mortinaissances. Pour juger de l'importance de cette lacune, il faut cependant rappeler que la majorité des IVG ne repose pas sur des raisons médicales. D'un autre côté, malgré I'utilisation d'une clé d'appariement dans la présente étude, certains doublons n'ont pu être évités, soit les enfants ayant changé de RSS de résidence entre deux hospitalisations ou ayant visité plus d'un centre hospitalier. La prévalence calculée des anomalies peut donc être légèrement surestimée.

Fait à noter, il a été décidé de dénombrer les cas jusqu'à cinq ans afin d'inclure les enfants diagnostiqués ou opérés tardivement. En effet, malgré une recommandation de correction chirurgicale avant 18 mois pour la cryptorchidie, I'âge moyen auquel elle est effectuée est de 38,5 mois selon une étude torontoise ${ }^{32}$. Cela pourrait en partie être dû aux cas de cryptorchidie acquise, soit par ré-ascension après une résolution spontanée, soit par l'ascension après la naissance'. Quant à l'hypospadias, la chirurgie n'est indiquée que si des répercussions fonctionnelles sont présentes ou prévisibles $^{33,34}$. Un sous-dénombrement pour cette anomalie peut donc être introduit par l'absence de données sur les consultations et les suivis en clinique externe.

Par ailleurs, si la prévalence d'hypospadias est stable entre 1989 et 2004, celle de la cryptorchidie est légèrement plus faible dans les périodes postérieures à 1995 comparati-

Tableau 2. Nombre de cas de cryptorchidie et d'hypospadias pour l'ensemble du Québec, 1989-2004

\begin{tabular}{|c|c|c|c|c|}
\hline \multirow[b]{2}{*}{ Périodes } & \multicolumn{2}{|c|}{ Cryptorchidie } & \multicolumn{2}{|c|}{ Hypospadias } \\
\hline & Nombre de cas & $\begin{array}{l}\text { Nombre moyen } \\
\text { de cas par année }\end{array}$ & Nombre de cas & $\begin{array}{c}\text { Nombre moyen } \\
\text { de cas par année }\end{array}$ \\
\hline $1989-90$ à 1991-92 & 2890 & 963,3 & 1659 & 553,0 \\
\hline $1992-93$ à 1994-95 & 2819 & 939,7 & 1605 & 535,0 \\
\hline $1995-96$ à 1997-98 & 2313 & 771,0 & 1445 & 481,7 \\
\hline $1998-99$ à 2000-01 & 2094 & 698,0 & 1223 & 407,7 \\
\hline 2001-02 à 2002-04 & 2090 & 696,7 & 1348 & 449,3 \\
\hline
\end{tabular}




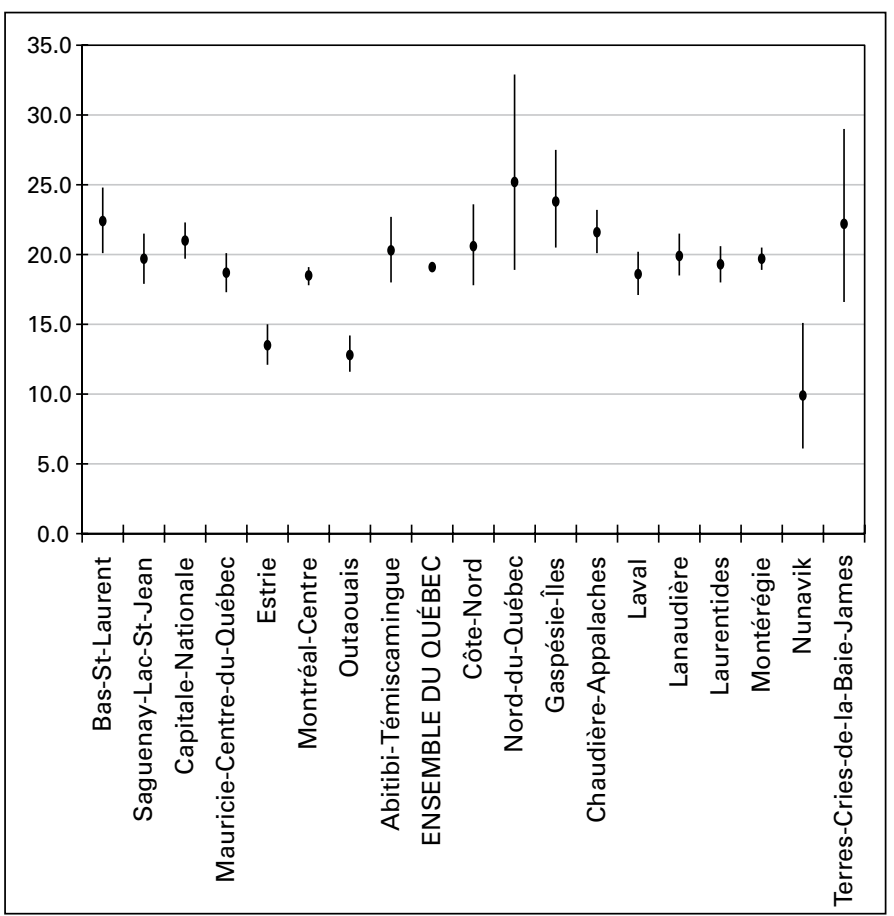

Graphique 2. Taux annuel moyen de cryptorchidie pour 1000 naissances vivantes de sexe masculin, par région sociosanitaire, Québec, 1989-2004.

vement à la première période de 1989-92. L'usage croissant de la classification CIM-10 à partir de 2001 pourrait théoriquement occasionner des différences avec la classification antérieure et influer sur la tendance ${ }^{31}$. Dans les faits, cette nouvelle classification n'est utilisée pour les hospitalisations au Québec que depuis 2006. II est possible également que la population à risque ait diminué. Depuis les années 60 , le dépistage prénatal des aneuploïdies est offert aux Québécoises et permet une interruption médicale de grossesse à celles la désirant ${ }^{35}$. Cette intervention, dont la pratique augmente depuis ses débuts, pourrait être à l'origine d'une diminution de la prévalence des maladies génétiques associées aux malformations urogénitales. D'autre part, les cas plus graves et plus évidents d'hypospadias ont une plus grande probabilité d'être inscrits sur la feuille sommaire que les cas légers et ainsi d'être comptabilisés dans MED-ÉCHO. Cette sous-représentation des cas légers pourrait masquer une tendance à la hausse puisque ce sont ces derniers qui sont en augmentation selon certains auteurs ${ }^{15}$.

Enfin, on observe une prévalence accrue ou réduite de cryptorchidie et/ou d'hypospadias notés à la naissance dans certaines régions du Québec. Contre toute attente, les taux plus élevés de cryptorchidie ne se retrouvent pas nécessairement dans les régions où une alimentation de subsistance a été associée à une surexposition aux contaminants de la chaîne alimentaire comme dans les régions nordiques ou côtières ${ }^{36,37}$. En effet, parmi les régions du Nunavik, des Terres-Cries-de-la-Baie-James, du Nord-du-Québec, de la
Côte-Nord et de la Gaspésie-îles-de-la-Madeleine, seule cette dernière présente une prévalence supérieure à celle du Québec pour la cryptorchidie. La Gaspésie-Îles-de-laMadeleine est d'ailleurs une des deux seules régions, avec la Capitale-Nationale, à présenter des taux en excès pour les deux anomalies fusionnées (données non présentées). Bien que ces résultats n'écartent pas l'hypothèse environnementale, ils portent à croire que d'autres facteurs contribueraient à expliquer une telle variation géographique.

Il importe toutefois de souligner les forces de cette étude. En effet, elle est la première s'intéressant à la situation actuelle de la cryptorchidie et de l'hypospadias au Québec. Aussi, la base de données utilisée lors de la comptabilisation de cas regroupait tous les hôpitaux du Québec avec un système de codification standardisé, ce qui a permis une uniformisation des résultats tout en assurant la couverture de l'ensemble des régions québécoises.

Afin de réduire le biais et d'augmenter la puissance d'une éventuelle étude, un système de surveillance québécois avec dépistage systématique et standardisé chez les nouveau-nés des anomalies congénitales devrait être instauré à l'instar du «Congenital Anomalies Reporting Form » en Alberta et du "Congenital Anomalies Surveillance Programme » en Colombie-Britanique ${ }^{31}$. Ceci fournirait des données plus justes sur ces anomalies, incluant les mortinaissances et les IVG, et colligerait les facteurs de risques maternels. En attendant, la validité du fichier MED-ÉCHO pourrait être évaluée en étudiant la concordance entre le dossier des patients et

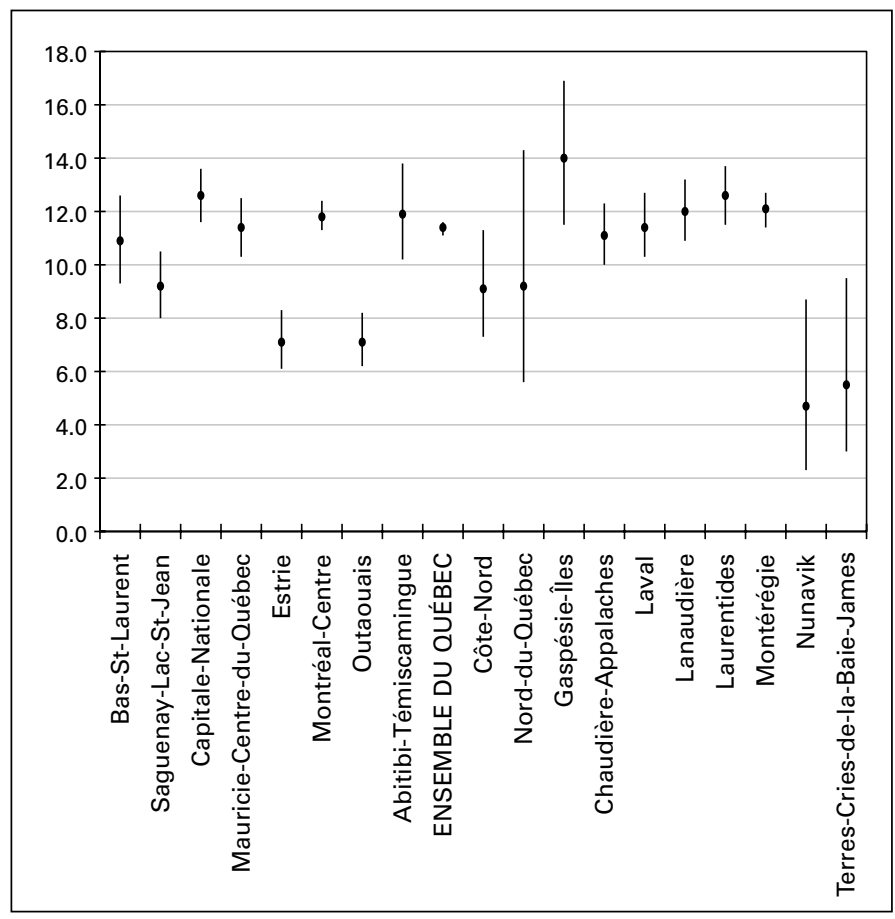

Graphique 3. Taux annuel moyen d'hypospadias pour 1000 naissances vivantes de sexe masculin, par région sociosanitaire, Québec, 1989-2004. 
les cas rapportés dans MED-ÉCHO pour la cryptorchidie et I'hypospadias.

\section{Conclusion}

Règle générale, entre 1989 et 2004, la prévalence de la cryptorchidie a connu une légère diminution alors que celle de l'hypospadias est demeurée stable. Comparativement à l'échelle de la province, des variations régionales significatives sont observables. D'autres études sont nécessaires afin $d^{\prime}$ 'évaluer l'hypothèse émergente $d^{\prime}$ 'un lien avec les contaminants environnementaux. L'implantation d'un système de surveillance plus adéquat contribuerait grandement à obtenir un portrait plus juste des anomalies congénitales.

Remerciements : Les auteurs tiennent à remercier Michel Carbonneau et Janie Boucher respectivement pour le soutien méthodologique et la compilation des données, Manon Blackburn pour les spécifications sur les biais, Pascale Côté pour son aide au niveau de la recherche d'articles et Marie-Cécile Gladel pour son support constant.

Competing interests: None declared.

This paper has been peer-reviewed.

\section{Bibliographie}

1. Virtanen HE, Toppari J. Epidemiology and pathogenesis of cryptorchidism. Hum Reprod Update 2008; 1:4958.

2. Olesen I, Sonne B, Hoei-Hansen C, et al. Environment, testicular dysgenesis and carcinoma in situ testis. Best Pract Res Clin Endocrinol Metab 2007;21:462-78.

3. Akre 0 , Boyd $H A$, Ahlgren $M$, et al. Maternal and gestational risk factors for hypospadias. Environ Health Perspect 2008;116:1071-6.

4. Bay K, Asklund C, Skakkebaek NE, et al. Testicular dysgenesis syndrome: possible role of endocrine disrupters. Best Pract Res Clin Endocrinol Metab 2006;20:77-90.

5. Damgaard IN, Jensen TK, Skakkebaek, et al. Risk factors for congenital cryptorchidism in a propective birth cohort study. PloS One 2008;3:e3051.

6. Pierik FH, Burdorf A, Deddens JA, et al. Maternal and paternal risk factors for cryptorchidism and hypospadias: a case-control study in newborn boys. Environ Health Perspect 2004;112:1570-6.

7. Møller H, Weidner SI. Epidemiology of cryptorchidism and hypospadias. Epidemiology 1999;10:352-3.

8. Akre 0 , Lipworth L, Cnattingius S, et al. Risk factor patterns for cryptorchidism and hypospadias. Epidemiology 1999;10:364-9.

9. Damgaard IN, Jensen TK, Petersen JH, et al. Cryptorchidism and maternal alcohol consumption during pregnancy. Environ Health Perspect 2007;115:272-7.

10. Weidner IS, Møller H, Jensen TK, et al. Risk factors for cryptorchidism and hypospadias. J Urol 1999;161:1606-9.

11. Virtanen HE, Tapanainen AE, Kaleva MM, et al. Mild gestational diabetes as a risk factor for congenital cryptorchidism. J Clin Endocrinol Metab 2006;91:4862-5.

12. Porter MP, Faizan MK, Grady RW, Mueller BA. Hypospadias in Washington State: maternal risk factors and prevalence trends. Pediatrics 2005;115:e495-9.

13. Toparri J, Skakkebaek NE. Sexual differentiation and environmental endocrine disrupters. Baillieres Clin Endocrinol Metab 1998;12:143-56.
14. Paulozzi L. International trends in rates of hypospadias and cryptorchidism. Environ Health Perspect 1999;107:297-302.

15. Toppari J, Kaleva M, Virtanen HE. Trends in the incidence of cryptorchidism and hypospadias, and methodological limitations of registry-based data. Hum Reprod Update 2001;7:282-6.

16. Toppari J, Larsen JC, Christiansen P, et al. Male reproductive health and environmental xenoestrogens. Environ Health Perspect 1996;104(Suppl 4):741-803.

17. Damgaard NI, Main KM, Toppari J, et al. Impact of exposure to endocrine disrupters in utero and in childhood on adult reproduction. Best Pract Res Clin Endocrinol Metab 2002;16:289-309.

18. Mauduit $C$, Florin $A, A$ amara $S$, et al. Effets à long terme des perturbateurs endocriniens environnementaux sur la fertilité masculine. Gynecol Obstet Fertil 2006;44:978-84.

19. Fernandez $M, 0 \mid$ mos $B$, Olea N. Exposicion a disruptores endocrinos y alteraciones del tracto urogenital masculino (criptorquidia e hipospadias). Gac Sanit 2007;21:500-14.

20. Sharpe R, Skakkebaek NE. Testicular dysgenesis syndrome: mechanistic insights and potential new downstream effects. Fertil Steril 2008;89(Suppl 1):e33-8.

21. Skakkebaek NE, Rajpert-de Meyts E, Main KM. Testicular dysgenesis syndrome: an increasingly common developmental disorder with environmental aspects. Hum Reprod 2001;16:972-8.

22. Wohlfahrt-Veje C, Main KM, Skakkebaek N. Testicular dysgenesis syndrome; Fetal origin of adult reproductive problems. Clin Endocrinol (Oxf) 2009;71:459-65.

23. Fernandez MF, Olmos B, Granada $A$, et al. Human exposure to endocrine-disrupting chemicals and prenatal risk factors for cryptorchidism and hypospadias: A nested case-control study. Environ Health Perspect 2007;115(Suppl 1):8-14.

24. Damgaard IN, Skakkebaek NE, Jensen TK, et al. Persistent pesticides in human breast milk and cryptorchidism. Environ Health Perspect 2006;114:1133-8.

25. Weidner $\mathrm{SI}$, Moller H, Jensen TK, et al. Cryptorchidism and hypospadias in sons of gardeners and farmers. Environ Health Perspect 1998; 106:793-6.

26. Parks LG, Ostby IS, Lambright CR, et al. The plasticizer diethylhexyl phthalate induces malformations by decreasing fetal testosterone synthesis during sexual differentiation in the male rat. Toxicol Sci 2000:58:339-49.

27. Main KM, Kiviranta H, Virtanen HE, et al. Flame retardants in Placenta and Breast Milk and Crytorchidism in Newborn Boys. Environ Health Persp 2007;115:1519-26.

28. Beaucage C, Bonnier-Viger Y. Épidémiologie appliquée : une initiation à la lecture critique de la littérature en sciences de la santé. Montréal, QC : Gaëtan Morin; $1996: 216$.

29. Institut de la statistique du Québec. Juin 2009. http://www.stat.gouv.qc.ca/. Accessed April 18, 2011.

30. INSPQ, MSSSQ et ISQ. Portrait de santé du Québec et de ses régions 2006 : les statistiques-Deuxième rapport national sur l'état de santé de la population du Québec. Gouvernement du Québec, QC : 2006; 270.

31. Annual Report 2007 with data for 2005: International Clearinghouse for Birth Defects Surveillance and Research. The Centre of the International Clearinghouse for Birth Defects Surveillance and Research. Roma, Italia; 2007. http://www.icbdsr.org/filebank/documents/ar2005/Report2007.pdf. Accessed april 18, 2011.

32. Merguerian PA, Mevorach RA, Shortliffe LD, et al. Laparascopy for the evaluation and management of the nonpalpable testicle. Urology 1998;51 (5A Suppl):3-6.

33. Kliegman RM, Marcdante KJ, Jenson HB, et al. Nelson Essentials of Pediatrics. 5th ed, Philadelphia, PA: Elseiver Saunders; 2006:1008.

34. Turgeon J, Bernard-Bonnin AC, Gervais P, et al. Dictionnaire de thérapeutique pédiatrique Weber. 2e éd, Montréal, QC: Gaëtan Morin; 2008:652.

35. Summers AM, Langlois $S$, Wyatt $P$, et al. Directive Clinique de la SOGC: Dépistage prenatal de l'aneuplö̈die foetale. SOGC 2007;187. wWw.sogc.org/guidelines/documents/187F-CPG-February2007.pdf. Accessed April 18, 2011.

36. Muckle G, Ayotte P, Dewailly EE, et al. Prenatal exposure of the northern Québec Inuit infants to environmental contaminants. Environ Health Perspect 2001;109:1291-9.

37. Sandau $C D$, Ayotte P, Dewailly E, et al. Pentachlorophenol and hydroxylated polychlorinated biphenyl metabolites in umbilical cord plasma of neonates from coastal populations in Québec. Environ Health Perspect 2002;110:411-7.

Correspondence: Dr Fabien Gagnon, Professeur d'enseignement clinique, Département des sciences de la santé communautaire, Faculté de médecine et des sciences de la santé, $3001,12^{e}$ Avenue Nord, Sherbrooke (Québec), Canada, J1H 5N4, fax : 001-819-564-5397; Fabien.Gagnon@ USherbrooke.ca 